\title{
Branch-imbalance relaxation times in superconductors
}

\author{
John Clarke, ${ }^{*}$ Ulrich Eckern, Albert Schmid, Gerd Schön, and Michael Tinkham ${ }^{\dagger}$ \\ Institut für Theorie der Kondensierten Materie, Universität Karlsruhe, 7500 Karlsruhe, Germany
}

(Received 12 February 1979)

\begin{abstract}
The interrelationship of $\tau_{Q}, \tau_{Q}^{*}$, and $\tau_{R}$, all of which have been used by various authors to describe the relaxation of branch imbalance, is critically reviewed with emphasis on the tractable case of disequilibrium generated by low-voltage tunnel injection and on the physical basis for the differences among the various times.
\end{abstract}

A tunneling current from a normal metal into a superconducting film generates a potential difference $V$. between pairs and quasiparticles in the superconductor. ${ }^{1}$ The quasiparticle potential can be sensed by a second normal-metal film coupled via a tunnel barrier to the reverse side of the superconductor. Since the pair chemical potential remains constant throughout the superconductor, $V$ is conveniently measured relative to a second probe (normal or superconducting, tunneling or metallic) coupled to the superconductor at a point far from the injection region. Tinkham and Clarke ${ }^{2}$ (TC) and Tinkham ${ }^{3}$ showed that

$$
V=Q^{*} / 2 e N(0) g_{N S}(T) \text {. }
$$

Here, $g_{N S}(T)$ is the measured normalized conductance of the probe junction in the limit $e V \ll<k_{B} T$, and $N(0)$ is the density of states for electrons of one spin. The quantity $e Q^{*}$ is the net quasiparticle charge per unit volume, with

$$
\begin{aligned}
Q^{*} & =\sum_{k} f_{k} q_{k} \\
& =2 N(0) \int_{\Delta}^{\infty}\left(f_{k}-f_{k_{<}}\right) d E_{k},
\end{aligned}
$$

where $f_{k}$ is the occupation number of the state $k, k_{>}$ and $k_{<}$refer to states of energy $E_{k}=\left(\Delta^{2}+\epsilon_{k}^{2}\right)^{1 / 2}$ with $k>k_{F}$ and $k<k_{F}$, respectively, and

$$
\begin{aligned}
q_{k} & \equiv \epsilon_{k} / E_{K}=u_{k}^{2}-v_{k}^{2} \\
& = \pm\left(E_{k}^{2}-\Delta^{2}\right)^{1 / 2} / E_{k}= \pm N_{s}^{-1}\left(E_{k}\right)
\end{aligned}
$$

is the effective charge of a quasiparticle in the state $k$. In Eq. (3),

$$
u_{k}^{2}\left(\epsilon_{k}\right)=v_{k}^{2}\left(-\epsilon_{k}\right)=\frac{1}{2}\left(1+\epsilon_{k} / E_{k}\right),
$$

and $N_{s}\left(E_{k}\right)$ is the normalized BCS density of states. (Here, and throughout the paper, we neglect all rounding of the BCS density of states.) Thus, Eq. (1) implies that $Q^{*}$ can be considered to be a directly measurable quantity, apart from the usual material parameter $N(0)$.

One wishes to interpret measured values of $Q^{*}\left(I_{\mathrm{inj}}, V_{\mathrm{inj}}, T\right)$ in terms of an injection rate and an appropriate relaxation time. In their original work,
TC considered an injection rate $\dot{Q}_{\text {inj }}$ and relaxation time $\tau_{Q}$ of the quasiparticle number branch imbalance $Q$ defined by

$$
\begin{aligned}
Q & =\sum_{k_{>}} f_{k}-\sum_{k_{<}} f_{k}< \\
& =2 N(0) \int_{\Delta}^{\infty} N_{s}\left(E_{k}\right)\left(f_{k}-f_{k_{<}}\right) d E_{k},
\end{aligned}
$$

which differs from $Q^{*}$ in that it does not take account of the fractional effective charges. Subsequently, Schmid and Schön ${ }^{4}$ (SS), and, later still, Eckern and Schön ${ }^{5}$ (ES) calculated these (and other) effects using a description that involves electrons rather than quasiparticles. In this picture, the total injected current (without regard to its quasiparticle composition) is taken as the source of the effect, and the characteristic time $\tau_{R}$ is proportional to the ratio of $V$ to this total current. More recently, Pethick and Smith $^{6}$ (PS) introduced an alternative approach using the quasiparticle description in which they consider the injection rate $\dot{Q}_{\text {inj }}^{*}$ and relaxation time $\tau_{q}=\tau_{Q^{*}}$ of the charge imbalance $Q^{*}$.

The purpose of this Comment is to make completely explicit the interrelationship among $\tau_{Q}, \tau_{Q^{*}}$, and $\tau_{R}$, and their relation to experimentally measureable quantities. This is done by drawing together results from the literature, and discussing in parallel the physical basis for the different approaches. We conclude, in agreement with PS, that $\tau_{Q^{*}}$ is a more appropriate time than $\tau_{Q}$ and should supersede it, whereas $\tau_{R}$ reflects a different conceptual point of view. For clarity, we confine almost the entire discussion to the analytically tractable case of lowvoltage injection, where $e V_{\text {inj }} \ll k_{B} T$. We also comment on the relation between the measured relaxation times and the quasiparticle lifetime.

The three schemes outlined above (respectively SS, PS, and TC) can be expressed as follows:

$$
\begin{aligned}
& Q^{*}=\left(I_{\mathrm{inj}} / e \Omega\right) \tau_{R}, \\
& Q^{*}=\dot{Q}_{\mathrm{inj}}^{*} \tau_{Q^{*}}, \\
& Q^{*}=\left(Q^{*} / Q\right) \dot{Q}_{\mathrm{inj}} \tau_{Q},
\end{aligned}
$$

and 
where $\Omega$ is the volume of the nonequilibrium region, and we have identified the $\delta Q_{n}^{\text {l.e. }}$ of PS with $Q^{*}$.

In Eq. (5), $I_{\text {inj }}$ is the total electric current injected

$$
\begin{aligned}
I_{\mathrm{inj}}=\frac{G_{N N}}{e} \int_{\Delta}^{\infty} N_{s}(E)\left[f\left(E-e V_{\mathrm{inj}}\right)\right. & \left.-f\left(E+e V_{\mathrm{inj}}\right)\right] d E \\
& \rightarrow G_{N N} V_{\mathrm{inj}}\left[2 \int_{\Delta}^{\infty}\left[-\frac{\partial f}{\partial E}\right) N_{s}(E) d E\right] \\
& \equiv G_{N N} V_{\mathrm{inj}} Y(T) \quad\left(e V_{\mathrm{inj}}<<k_{B} T\right) .
\end{aligned}
$$

Here, $G_{N N}$ is the tunnel conductance with both metals in the normal state. Note that the Yosida ${ }^{7}$ function $Y(T)$ is the same as $g_{N S}$ for the ideal BCS case. We use the notation $Y$ to avoid confusion with the measured $g_{N S}$ of the probe junction which appears in Eq. (1).

In Eq. (6), $\dot{Q}_{\text {inj }}^{*}$ is the rate injection of net quasiparticle charge, shown by Pethick and Smith to be

$$
\begin{aligned}
& \dot{Q}_{\mathrm{inj}}^{*}= \frac{G_{N N}}{e^{2} \Omega} \int_{\Delta}^{\infty} N_{s}^{-1}(E)\left[f\left(E-e V_{\mathrm{inj}}\right)\right. \\
&\left.-f\left(E+e V_{\mathrm{inj}}\right)\right] d E \\
& \rightarrow \frac{G_{N N} V_{\mathrm{inj}}}{e \Omega}\left[2 \int_{\Delta}^{\infty}\left[-\frac{\partial f}{\partial E}\right) N_{s}^{-1}(E) d E\right] \\
& \equiv \frac{G_{N N} V_{\mathrm{inj}}}{e \Omega} Z(T)=\frac{Z}{Y} \frac{I_{\mathrm{inj}}}{e \Omega} \\
&\left(e V_{\mathrm{inj}}<<k_{B} T\right)
\end{aligned}
$$

Note that this $Z(T)$ is identical with the $f(T)$ of ${ }^{3} \mathrm{He}$ literature ${ }^{8}$ but we prefer a different notation to avoid confusion with the Fermi function.

In Eq. (7), $\dot{Q}_{\text {inj }}$ is the rate of injection of branch imbalance, found by Tinkham and Clarke ${ }^{2}$ to be

$$
\begin{aligned}
\dot{Q}_{\mathrm{inj}}= & \frac{g_{N N}}{e^{2} \Omega} \int_{\Delta}^{\infty}\left[f\left(E-e V_{\mathrm{inj}}\right)\right. \\
& \left.-f\left(E+e V_{\mathrm{inj}}\right)\right] d E \\
\rightarrow & \frac{G_{N N} V_{\mathrm{inj}}}{e \Omega}\left[2 \int_{\Delta}^{\infty}\left[-\frac{\partial f}{\partial E}\right] d E\right] \\
\equiv & \frac{G_{N N} V_{\mathrm{inj}}}{e \Omega}[2 f(\Delta)] \\
= & \frac{2 f(\Delta)}{Y} \frac{I_{\mathrm{inj}}}{e \Omega} \quad\left(e V_{\mathrm{inj}}<<k_{B} T\right) .
\end{aligned}
$$

The reasons for considering the several schemes can be summarized as follows. Equation (5) has the advantage of defining $\tau_{R}$ in terms of a strictly measurable quantity $\zeta \equiv \Omega g_{N S} V / I_{\text {inj. }}$ Equation (6) deals most straightforwardly with the central quantity $Q^{*}$. Equation (7) is appropriate if one focuses on the branch imbalance $Q$ rather than the fractionalcharge-weighted imbalance $Q^{*}$. Historically, Eq. (7) was the first approach used, ${ }^{1-3}$ but here we support the proposal of PS that it be superseded by Eq. (6).

We consider in detail only the case of low injection voltage, $e V_{\text {inj }}<<k_{B} T$, where the response $Q^{*}$ (i.e., $V$ ) is linear in $I_{\mathrm{inj}}$, and can be calculated explicitly. Then, the consistency of Eqs. (5), (6), and (7) requires the following relations between the various relaxation times and the measured quantity $\zeta$ :

$$
\begin{aligned}
\tau_{R} & =\frac{Z}{Y} \tau_{Q^{*}}=\frac{Q^{*}}{Q} \frac{2 f(\Delta)}{\dot{Y}} \tau_{Q} \\
& =\frac{e \Omega Q^{*}}{I_{\text {inj }}}=2 e^{2} N(0)\left(\frac{\Omega g_{N S} V}{I_{\text {inj }}}\right) \\
& \equiv 2 e^{2} N(0) \zeta \quad\left(e V_{\text {inj }}<<k_{B} T\right) .
\end{aligned}
$$

[At higher injection voltages, the factors relating the times would have to be replaced by the more general forms from which they were derived, namely $Z / Y$ by $e \Omega \dot{Q}_{\mathrm{inj}}^{*} / I_{\text {inj }}$ and $2 f(\Delta) / Y$ by $e \Omega \dot{Q}_{\mathrm{inj}} / I_{\text {inj. }}$.] The ratio $Z / Y$, which determines $\tau_{R} / \tau_{Q^{*}}$ for this low-voltage injection case, is a well-defined function of $T$, which reduces to 1 at $T_{c}$ and to $k_{B} T / \Delta$ at low temperatures (see Appendix). It is plotted in Fig. 1.

To find the relation of $\tau_{Q}$ to $\tau_{Q^{*}}$ and $\tau_{R}$, one must obtain a value for $Q^{*} / Q$, which depends on the distribution of nonequilibrium quasiparticles. For the present case of low-voltage injection, and especially near $T_{c}$ where $\tau_{R} \approx \tau_{Q^{*}} \approx \tau_{Q} \gg \tau_{E}$, it seems plausi-

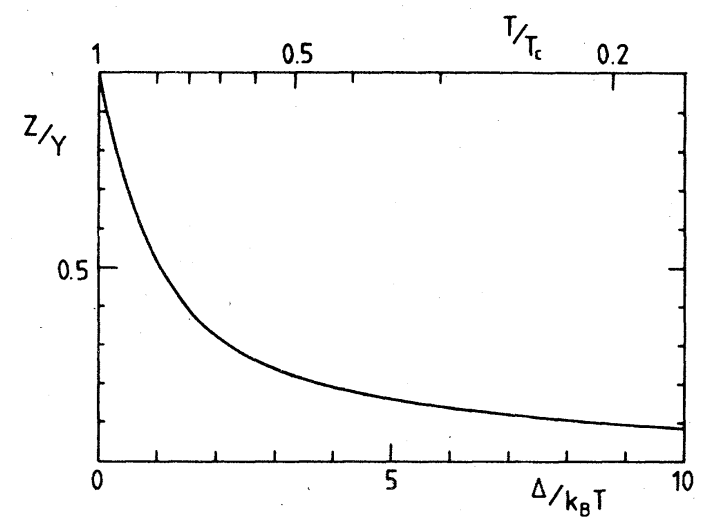

FIG. 1. Ratio $Z / Y$ vs $\Delta / k_{B} T$. From Eq. (11), $Z / Y=\tau_{R} / \tau_{Q^{*}}$, for low injection voltages. The quantities $Y$ and $Z$ are defined in Eqs. (8b) and (9b), and discussed further in the Appendix. 
ble that the energy distributions should be characterized by Fermi distributions with shifted chemical potentials. In the original Tinkham-Clarke work, it was assumed that the two branches of the quasiparticle spectrum were described by independent chemical potentials, such that $E_{k}$ was measured relative to $\mu_{>}$ and $\mu_{<}$for $k>k_{F}$ and $k<k_{F}$, respectively. In that case, it follows that

$$
\delta f_{>}-\delta f_{<}=\left(-\frac{\partial f}{\partial E}\right)\left(\mu_{>}-\mu_{<}\right) .
$$

With this form for $\left(\delta f_{>}-\delta f_{<}\right)$, it is easy to show that $Q^{*} / Q=2 f(\Delta) / Y$. Thus for $e V_{\text {inj }}<<k_{B} T$, one finds $\tau_{Q} / \tau_{Q^{*}}=Y(T) Z(T) /[2 f(\Delta)]^{2}$, which ranges from 1 at $T=T_{c}$ to $\frac{1}{2} \pi$ at $T=0$. However, in the more recent work of PS, it is argued that it is more physically reasonable to measure $\epsilon_{k}$ (rather than $E_{k}$ ) for both $k_{>}$and $k_{<}$states relative to a single shifted chemical potential $\mu$, so that $E=\left[\Delta^{2}+(\epsilon-\delta \mu)^{2}\right]^{1 / 2}$, where $\delta \mu$ is the change of $\mu$ relative to the pair chemical potential $\mu_{s}$. This causes a change in occupation number

$$
\delta f=\frac{\partial f}{\partial E} \frac{\partial E}{\partial \mu} \delta \mu=\left(-\frac{\partial f}{\partial E}\right) \frac{\epsilon}{E} \delta \mu
$$

so that

$$
\delta f_{>}-\delta f_{<}=2\left(-\frac{\partial f}{\partial E}\right) \frac{|\epsilon|}{E} \delta \mu .
$$

In Eq. (13), we have replaced $|\epsilon-\delta \mu|$ by $|\epsilon|$, since $\delta \mu$ is very small. A distribution of nonequilibrium electrons similar to Eq. (13) is also found in the work of Schmid and Schön, and in the numerical solution to the kinetic equation reported by $\mathrm{Chi}$ and Clarke ${ }^{9}$ for the case of high-voltage injection. [Note that $\delta f_{E}$ of SS is $(\epsilon / E) \delta f$ in our notation.] With the form given in Eq. (13), one finds $Q^{*} / Q=Z(T) / 2 f(\Delta)$, so that, from Eq. (11), at least in this limit of lowvoltage injection, $\tau_{Q}=\tau_{Q^{*}}$ for all temperatures. ${ }^{10}$

In the more general case of $e V_{\text {inj }} \geq k_{B} T$ and $\Delta \geq k_{B} T$, there is no simple expression for $Q^{*} / Q$, although the computer result of $\mathrm{Chi}$ and Clarke supports the notion that $Z(T) / 2 f(\Delta)$ may be a good approximation quite generally. In view of this uncertainty, and because the case for using Eq. (13) seems persuasive, it seems preferable to simply abandon the $\tau_{Q}$ scheme in favor of the $\tau_{Q^{*}}$ scheme, as was suggested by Pethick and Smith, and hereafter to concentrate on a comparison of the $\tau_{Q^{*}}$ and $\tau_{R}$ approaches, where a real difference of viewpoint exists.

We have already seen that near $T_{c}$, where $\Delta \ll k_{B} T$, the difference between $I_{\text {inj }} / e \Omega$ and $\dot{Q}_{\text {inj }}^{*}$ and hence between $\tau_{R}$ and $\tau_{Q^{*}}$ is small. At lower temperatures the factor $\epsilon^{2} / E^{2}$, which is different in the expressions for $\dot{Q}_{\text {inj }}^{*}$ [Eq. (9b)] and $I_{\text {inj }} / e \Omega$ [Eq.

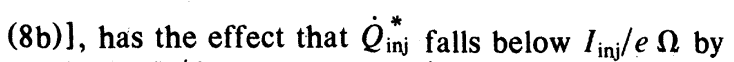
the factor $Z / Y$ plotted in Fig. 1. At low temperatures this ratio becomes $\approx k_{B} T / \Delta$ (see Appendix). As a result, the inferred value of $\tau_{R}$ will be a factor $Z / Y$ lower than the inferred value of $\tau_{Q^{*}}$ for the same experimental data. This difference in definiton of $\tau_{R}$ and $\tau_{Q^{*}}$ stems from the conceptual difference in the description used by TC and PS compared to that used by SS. In the SS formulation, the entire injection current $I_{\text {inj }}$ appears as a source for a quasiparticle charge (no factor $\epsilon^{2} / E^{2}$ ). However, near the gap edge the charge is very rapidly converted into a supercurrent at a rate [Eq. (28) of Ref. 5 ]

$$
\frac{1}{\tau_{\text {conv. }}}=\frac{2 \Delta N_{2}}{N_{1}} \approx \frac{\Delta^{2}}{\epsilon^{2}} \frac{1}{\tau_{E}},
$$

which diverges at the gap. (The notation here follows SS and ES. $N_{1}$ is the density of states and $N_{2}$ describes the quasiparticle-pair conversion as indicated by this equation. For further details, the reader should consult the original papers. ${ }^{4,5,11}$ ] Adding to this term the "scattering out" rate $\approx 1 / \tau_{E}$, we find an energy-dependent rate $\left(E^{2} / \epsilon^{2}\right) / \tau_{E}$. Thus, while in the TC-PS picture the injection rate is reduced by a factor $\epsilon^{2} / E^{2}$, in the SS approach the conversion rate is enhanced over the electron-phonon scattering rate by a factor $E^{2} / \epsilon^{2}$. The resulting stationary quasiparticle distribution functions are the same in both approaches [Eq. (13)]. The difference seems to be mainly semantic, at least in the steady-state situation.

In practical experiments at low temperatures, the injection voltage is normally large compared with $\Delta / e$ in order that sufficient current be injected to give measurable branch imbalance voltages. In that case, both $I_{\text {inj }}$ and $\dot{Q}_{\text {inj }}^{*}$ become nonlinear functions of $V_{\text {inj }}$ as well as of $T$, and the inferred relation between $\tau_{R}$ and $\tau_{Q^{*}}$ also becomes a function of $V_{\text {inj }}$ as well as $T$. A particularly illuminating example is found in the work of Chi and Clarke, ${ }^{9}$ in which the injected current is provided by an $S^{\prime}-S$ tunnel junction. In that case, there is a step increase in $I_{\text {inj }}$ when $V_{\text {inj }}$ passes through $\left(\Delta+\Delta^{\prime}\right) / e$ but no corresponding change is observed in the measured probe voltage. Because the entire increase in injected current occurs at the gap edge, where the effective $\epsilon_{k} / E_{k}$ is zero, there is no change in $\dot{Q}_{\text {inj }}^{*}$ at the step, and hence the experimental result implies that $\tau_{Q^{*}}$ also holds constant through the step. By contrast, $\tau_{R}$ must decrease abruptly to reflect the constant observed $V$ in the face of an abrupt increase in $I_{\text {inj. }}$. This example shows that some gain in insight can be obtained by decomposing the Schmid-Schön $\tau_{R}$ into two factors as $\tau_{R}=F^{*} \tau_{Q^{*}} \equiv\left(e \Omega \dot{Q}_{\text {inj }}^{*} / I_{\text {inj }}\right) \tau_{Q^{*}}$, since the first factor is readily calculated and leaves a value for $\tau_{Q^{*}}$ which is apparently relatively stable with respect to changes in injection conditions. 
Having measured $\tau_{Q^{*}}$ or $\tau_{R}$, one would like to determine a value for the quasiparticle lifetime, $\tau_{E}(T)$, due to electron-phonon collisions. In general, the inverse lifetime for a quasiparticle of energy $E$ is the sum of the scattering and recombination rates

$$
\tau_{E}^{-1}(T)=\tau_{s}^{-1}(E, T)+\tau_{r}^{-1}(E, T)
$$

In particular, one is interested in the normal-state lifetime at the Fermi energy and at $T_{c}, \tau_{E-0}\left(T_{c}\right)$. We can also relate $\tau_{E-0}\left(T_{c}\right)$ to the time $\tau_{0}$ of Kaplan et $a .^{12}$ by using Eq. (18) of Ref. 12 and noting that, since $\tau_{s}^{-1}(0, T)=\tau_{r}^{-1}\left(0, T_{c}\right)$,

$$
\tau_{E-0}^{-1}\left(T_{c}\right)=7 \zeta(3) \tau_{0}^{-1}=8.4 \tau_{0}^{-1} .
$$

Very close to $T_{c}$, the relation between $\tau_{Q^{*}}$ or $\tau_{R}$ and $\tau_{E-0}\left(T_{c}\right)$ is practically independent of injection voltage $4,6,13$

$$
(Y / Z) \tau_{R}=\tau_{Q^{*}}=4 k_{B} T \tau_{E-0}\left(T_{c}\right) / \pi \Delta .
$$

At lower temperatures, the relation of a measured value of $\tau_{Q^{*}}$ or $\tau_{R}$ to $\tau_{E-\Delta}(T)$ depends on temperature and injection voltage, and, in general, must be determined by a computer calculation. Such a calculation ${ }^{13}$ indicates that Eq. (17) is accurate to within $\pm 15 \%$ for $e V_{\text {inj }} \leqslant 3 \Delta(T)$ and $\Delta \leqslant k_{B} T$, an accuracy that is probably adequate for most practical purposes. The computations have also been extended to lower temperatures, but we emphasize that, in general, elastic impurity scattering in an anisotropic superconductor will make a substantial contribution to $\tau_{R}^{-1}$ or $\tau_{Q}^{-1}$, particularly at low temperatures where it eventually dominates. Also, magnetic impurities or an external magnetic field, if present, can drastically reduce $\tau_{R}$. Since the results given in this paper pertain to the case of inelastic phonon scattering only, they can be used only in the absence of such magnetic pairbreaking perturbations, and at temperatures high enough that the elastic scattering-anisotropy mechanism is dominated by the inelastic phonon one. This consideration combined with the need for a reliable quantitative theory implies that, in most practical situations, one can obtain reliable estimates of $\tau_{E-0}\left(T_{c}\right)$ only from data taken in the limit $\Delta \leq k_{B} T^{14}$ If one bears in mind these limitations, the absence of phonon-trapping effects (which can be uncertain to a factor of $\sim 2$ ) gives this type of measurement a substantial advantage over measurements of effective recombination times or energy relaxation times as a means of determining $\tau_{E}$. Moreover, these measurements have intrinsic interest as a unique example of a type of transport phenomenon in which the inelastic scattering time is dominant, since (apart from gap anisotropy effects) elastic scattering is ineffective for relaxing branch imbalance.

\section{ACKNOWLEDGMENTS}

Two of us (J.C.) and (M.T.) would like to thank the members of the Institut für Theorie der Kondensierten Materie, Universität Karlsruhe, for their warm hospitality during the course of this work. The support of this work by the Deutsche

Forschungsgemeinschaft and by the U.S. National Science Foundation is gratefully acknowledged. One of us (J.C.) would like to thank the Guggenheim Foundation for financial support. One of us (M.T.) would like to thank the Alexander von Humboldt Foundation for financial support.

\section{APPENDIX}

We define two integrals, and give their asymptotic values:

$$
\begin{aligned}
Y(T) & =2 \int_{\Delta}^{\infty} N_{s}(E)\left(-\frac{\partial f}{\partial E}\right) d E \\
& \rightarrow 1-\frac{7 \zeta(3)}{4 \pi^{2}}\left(\frac{\Delta}{k_{B} T}\right)^{2} \cdots \quad\left(\Delta<<k_{B} T\right) \\
& \rightarrow\left(\frac{2 \pi \Delta}{k_{B} T}\right)^{1 / 2} e^{-\Delta / k_{B} T}\left(\Delta>>k_{B} T\right)
\end{aligned}
$$

and

$$
\begin{aligned}
Z(T) & =2 \int_{\Delta}^{\infty} N_{s}^{-1}(E)\left(-\frac{\partial f}{\partial E}\right) d E \\
& \rightarrow 1-\frac{\pi \Delta}{4 k_{B} T}+\frac{7 \zeta(3)}{4 \pi^{2}}\left(\frac{\Delta}{k_{B} T}\right)^{2} \cdots \quad\left(\Delta<<k_{B} T\right) \\
& \rightarrow \frac{k_{B} T}{\Delta} Y(T)\left(\Delta>>k_{B} T\right)
\end{aligned}
$$

where $\zeta(3)=1.202 \cdots$. Strictly speaking, $Y$ and $Z$ are functions of $\Delta / k T$. They become functions of $T$ if one takes the BCS form of $\Delta(T)$.
"On sabbatical leave from the Dept. of Phys., Univ. of Calif., and Materials and Molecular Res. Div. of the Lawrence Berkeley Lab., Berkeley, Calif. 94720.

${ }^{\dagger}$ On sabbatical leave from Dept. of Phys., Harvard Univ., Cambridge, Mass. 02138.
1J. Clarke, Phys. Rev. Lett. 28, 1363 (1972); J. Clarke and J. L. Paterson, J. Low Temp. Phys. 15, 491 (1974).

${ }^{2}$ M. Tinkham and J. Clarke, Phys. Rev. Lett. 28, 1366 (1972).

${ }^{3}$ M. Tinkham, Phys. Rev. B $\underline{6}, 1747$ (1972). 
${ }^{4}$ A. Schmid and G. Schön, J. Low Temp., Phys. 20, 207 (1975).

${ }^{5}$ U. Eckern and G. Schön, J. Low Temp. Phys. $\underline{32}, 821$ (1978).

${ }^{6}$ C. J. Pethick and H. Smith, Ann. Phys. (N.Y.) 119,133 (1979).

${ }^{7}$ K. Yosida, Phys. Rev. 110, 769 (1958).

${ }^{8}$ R. Combescot and H. Ebisawa, Phys. Rev. Lett. $\underline{33}, 810$ (1974).

${ }^{9}$ C.C. Chi and J. Clarke, Phys. Rev. B 19, 4495 (1979).

${ }^{10}$ Near equality of $\tau_{Q^{*}}$ and $\tau_{Q}$ is also found in the numerical calculation of Chi and Clarke. Chang [Phys. Rev. B 19 , 1420 (1979)] shows that near $T_{c}$ and for energies $E \gg k T$, the asymptotic energy-dependent rate $\tau_{Q^{-1}}^{-1}(E)$ exceeds $\tau_{Q}^{-1}(E)$ by a factor of $\frac{1}{2} \pi$. However, at low energies he finds that $\tau_{Q^{-}}^{-1}(E)$ is less than $\tau_{Q}^{-1}(E)$.

Averaged over the appropriate nonequilibrium distribution [Eq.(13)], this difference apparently cancels out. In this connection, we also make the following remark on the original calculation of $\tau_{Q}$ by Tinkham (Ref.3). After correcting the identification of the parameter $\alpha$ by a factor of 2 (as noted earlier by Kaplan et al.) so that $\tau_{E=0}\left(T_{c}\right)=\left(8.4 \alpha T_{c}^{3}\right)^{-1}$, the calculation of Ref. 3, which used the asymptotic form of $\tau_{Q}^{-1}(E)$ for high $E$, gives $\tau_{Q}=\left(2 T_{c} / \Delta\right) \tau_{E-0}\left(T_{c}\right)$. Since the asymptotic form of
$\tau-\frac{1}{Q^{(}(E)}$ is simply larger by a factor of $\frac{1}{2} \pi$, the calculational procedure of Ref. 3 gives $\tau_{Q^{*}}=\left(4 T_{c} / \pi \Delta\right) \tau_{E-0}\left(T_{c}\right)$, in precise agreement with the exact result of SS and others. Although this exact agreement for $\tau_{Q^{\bullet}}$ must be considered fortuitous, the plots of Chi and Clarke comparing the exact and asymptotic form of $\tau_{Q}^{-1}$ and $\tau_{Q}^{-1}$ make it clear that the asymptotic formula should be a good approximation near $T_{c}$ for $\tau_{Q^{-1}}(E)$, but should definitely underestimate $\tau_{Q}^{-1}(E)$ because of the singular behavior of the exact form near $\Delta$. This singular behavior does not arise with $\tau_{Q^{-1}}(E)$, since the use of fractional charges in the definition of $Q^{*}$ eliminates the discontinuous structure of $Q$ at $\Delta$.

${ }^{11}$ The connection between the Green's function technique (SS) and the Boltzmann-equation approach (PS) is also discussed by O. Entin-Wohlman and R. Orbach, Phys. Rev. B 19,4510 (1979).

${ }^{12}$ S. B. Kaplan, C. C. Chi, D. N. Langenberg, J. J. Chang, S. Jafarey, and D. J. Scalapino, Phys. Rev. B $\underline{14}, 4854$ (1976).

${ }^{13}$ C. C. Chi and J. Clarke, Phys. Rev. B (to be published).

${ }^{14} \mathrm{However}$, in the case of $\mathrm{Al}$, where $\tau_{E-0}\left(T_{c}\right)$ is especially long, it is necessary to be much closer to $T_{c}$ for the inelastic scattering to dominate (Ref. 9). 